\title{
Hernia sac of indirect inguinal hernia: Invagination, excision or ligation?
}

\author{
Ibrahim Othman, MD; Hamdy Abdel Hady, MD
}

\author{
Department of General Surgery, Tanta University, Tanta, Egypt.
}

This study compares the effect of invaginating, excision of hernia sac without ligation with the traditional method of high ligation of the hernia sac on post-operative pain and recurrence.

Patients and methods: This multicenter prospective randomized study included 152 patients with 167 primary indirect inguinal hernias. In group I (54 hernias) the sac was not opened and was inverted with the finger into the peritoneal cavity. In group E (56 hernias) the sac was excised at the neck without ligation. In group L (57 hernias) the sac was transfixed at the neck and excised in the traditional manner. The repair of the posterior wall of the inguinal canal was done according to Lichtenstein tension free technique. Mean length of follow up was $81.50 \pm$ 22.34, $79.35 \pm 26.76$ and $77.83 \pm 21.26$ months respectively.

Results: Postoperative seroma occurred in 1 patient (0.60\%) in group E and 1 patient (0.60\%) in group L. Surgical site infection occurred in 2 patients (1.20\%) in group I, 1 patient $(0.60 \%)$ in group E and 2 patients (1.20\%) in group L. Mean postoperative pain score was $3.04 \pm 2.11$, $3.98 \pm 2.33$ and $4.06 \pm 2.43$ respectively (p: 0.049). Chronic pain occurred in 3 patients in group I (1.80\%), 3 patients in group E (1.80\%) and 5 patient in group L (3.00\%) (P: 0.749). The difference between the complications in three groups was statistically insignificant ( $p: 0.887)$. Hernia recurrence occurred in 3 patients (1.80\%) in group I, 1 patient $(0.60 \%)$ in group $E$ and 1 patient (0.60\%) in group L (p: 0.429).

Conclusion: Invagination and excision of the hernia sac do not have adverse effects on repair integrity. They limit the dissection and reduce the morbidity and risk of injury to the spermatic cord and surrounded structures. They are safer and more appropriate for repair of sliding hernia. Ligation of the hernia sac in inguinal hernia surgery is not only unnecessary and time consuming but also leads to increased postoperative pain. Recurrence rates are statistically unaffected by not ligating the sac.

Key words: Hernia sac, indirect inguinal hernia, invagination, excision, ligation

\section{Introduction:}

Hernia and its treatment have fascinated surgeons of all latitude throughout the years of recorded medical history. From the beginning of modern anatomic hernia surgery when Edoardo Bassini recommended excision and high ligation of the indirect hernia sac in $1887,{ }^{1}$ few studies have examined the best way to manage the indirect hernia sac, but have generated no consensus. Nevertheless, these studies showed that management of the indirect hernia sac may influence the rate of hernia recurrence and the development of postoperative pain. ${ }^{2-5}$
The value of peritoneal closure in abdominal wounds has long been questioned. The peritoneum heals rapidly within 3-5 days leaving no adhesions in both large and small defects. ${ }^{6}$ It has also been proven that leaving the peritoneum open in closure is not associated with an increased wound failure rate. ${ }^{7}$

Recurrences have frustrated surgeons of all ages, experience, skill and nationality. One of the cardinal causes of recurrence was thought to be failure to high ligate the sac. However, the emphasis has now shifted from the sac to the defect and hence the necessity 
to ligate the sac has been questioned. In the modern operations for inguinal hernia like the Liechtenstein's repair and Rutkow's Mesh plug repair the sac is not ligated but simply inverted or excised without ligation.8,9

This study compares the effect of invaginating, excision of hernia sac without ligation with the traditional method of high ligation of the hernia sac on post-operative pain and recurrence.

\section{Patients and methods:}

This multicenter prospective randomized study was performed in General Surgery Department, Tanta University Hospitals, and Ghodran General Hospital, Kingdom of Saudi Arabia during the period from July 2003 to December 2012 on 152 patients with primary indirect inguinal hernias (167 hernias). Only indirect, uncomplicated hernias in adult patients were included. Recurrent hernias were excluded.

Full explanation of procedures, possible complications and patient consent were assured before inclusion in the research. The study protocol was approved by the Ethics Committee of General Surgery Department, Tanta University Hospitals.

Patients were randomly categorized into 3 groups through a computer randomization program.

All patients underwent detailed medical history, clinical examination and abdominal ultrasound examination in addition to routine hematological and biochemical investigations for preparation for anesthesia.

Patients were hospitalized on the day of surgery. All procedures were performed under spinal, general or local anesthesia. All patients received a single dose of Augmentin 1.2 grams (Amoxicillin and clavulinic acid, www.gsk.com) 30 minutes prior to surgery.

Inguinal canal was opened in the usual manner. The indirect sac was dissected up to the neck. In group I (from Invagination) (54 hernias) the sac was not opened and was inverted with the finger into the peritoneal cavity. In group E (from Excision) (56 hernias) the sac was excised at the neck without ligation; the residual defect in the parietal peritoneum was left unattended. In group L (from Ligation) (57 hernias) the sac was transfixed at the neck and excised in the traditional manner.

The repair of the posterior wall of the inguinal canal was done according to Lichtenstein tension free technique. A polypropylene mesh of $7.5 \times 15 \mathrm{~cm}$ (Prolene mesh, www.ecatalog.ethicon.com), was fashioned and placed with an overlap of the pubic bone. The mesh was fixed with a monofilament non absorbable suture. The ilioinguinal nerve and the iliohypogastric nerve were identified and spared. The external oblique fascia was closed with absorbable sutures.

Patients were allowed to eat and drink 3 hours after recovery. Analgesia was given in the form of $100 \mathrm{mg}$ Pethedine on recovery and another $100 \mathrm{mg}$ Pethedine intramuscularly after 12 hours if the patient complained of pain. Postoperative pain was measured for two weeks after surgery using pain visual analog scale (VAS) on daily base and collectively for the first 2 postoperative weeks. Patients were discharged from hospital in the first post-operative morning. Oral non steroidal anti-inflammatory analgesia were prescribed (Diclofinac potassium, Cataflam $50 \mathrm{mg}$ tablet TM. www.pharma.us.novartis.com) to be taken if the patient complained of pain. Patients were followed in visits at three day interval for two weeks then every 3 months. Patients that did not come for follow up were contacted by telephone and interviewed about chronic pain and recurrence. Any patient reporting recurrence in the operated groin was scheduled for an outpatient clinic check. Patients were encouraged to visit the clinic at any time if they had any problem. The total follow-up time was calculated based on the last visit to the outpatient clinic or the contact via telephone. The mean length of follow up was $81.50 \pm 22.34,79.35 \pm 26.76$ and 77.83 \pm 21.26 months respectively.

Primary complications included infections and formation of hematomas or seromas. A superficial infection was defined as an infection not requiring surgical treatment. A deep infection was defined as an infection 
at or near the site of hernia surgery that required surgical intervention. Hematoma and/or seroma formation was considered a primary complication even if it resorbed spontaneously. Late complications included recurrence and chronic pain.

\section{Statistical analysis:}

Quantitative variables were expressed as mean \pm SD. Qualitative variables were expressed as frequency and percent. Quantitative parametric variables were compared between the three groups using the ANOVA test, quantitative non-parametric variables were compared using MannWhitney test. Qualitative variables were compared using Chi-square test or Fisher exact test when the criteria for using Chisquare were not sufficient. The power used was 0.80 while the level of significance was $5 \%$.

\section{Results:}

The demographic characteristics and clinical data of group I (50 patients), group E (49 patients) and group L (53 patients) are summarized in Tables $(\mathbf{1 , 2})$.

Postoperative seroma did not occur in group I, occurred in 1 patient in group E $(0.60 \%)$ and 1 patient in group L (0.60\%). They were successfully treated conservatively.

Surgical site infection occurred in 2 patients in group I (1.20\%), 1 patient in group $\mathrm{E}(0.60 \%)$ and 2 patients in group L (1.20\%). Four patients $(2.40 \%)$ were managed by proper antibiotics according to culture and sensitivity result. One patient in group I $(0.60 \%)$ had deep infection that needed drainage of collection and leaving the wound open until pus formation stopped. Mesh removal was not necessary.

Mean postoperative pain score for first 2 weeks was $3.04 \pm 2.11$ in group I, 3.98 \pm 2.33 in group $\mathrm{E}$ and $4.06 \pm 2.43 \mathrm{n}$ group $\mathrm{L}$ (p: 0.049). Most patients improved after medical treatment with oral non steroidal anti-inflammatory analgesia. Pain persisted in 11 patients. They were considered to have chronic pain.

Chronic pain occurred in 3 patients in group I (1.80\%), 3 patients in group $\mathrm{E}$ (1.80\%) and 5 patient in group L (3.00\%) (P: 0.749). Four patients (2.40\%) markedly improved after medical treatment. Others suffered from persistent low grade pain that partially improved (5 patients, $3.00 \%$ ) or failed to improve until the end of study (1 patient, $0.60 \%$ ).

The difference between the complications in the three groups was statistically insignificant (p: 0.887).

\section{Recurrence:}

Hernia recurrence occurred in 3 patients in group I (1.80\%), 1 patient in group $\mathrm{E}$ $(0.60 \%)$ and 1 patient in group L $(0.60 \%)$. In group I recurrence occurred 23, 35 and 42 months after operation, in group E recurrence occurred 34 months after operation while in group $\mathrm{L}$ recurrence occurred 24 months after operation. Patients were treated by either Lichtenstein or TEP Laparoscopic Hernioplasty. No rerecurrence happened to them until the end of this study. The difference between the recurrence rates in the three groups was statistically insignificant (p: 0.429). Post-operative complications are summarized in Table (4).

\section{Discussion:}

Surgeons have long undertaken the burden of the 'sac' in inguinal hernia repair. Thus the sac got pride of place in hernia surgery at the expense of the 'defect'. It is a long held belief that ligating the sac is an important adjunct to groin hernia operations. 9,10

Various reports discussing recurrence considered excision and high ligation of the indirect inguinal hernia sac an essential part of the repair, and if not performed properly recurrence is more common. ${ }^{11-14}$ Studies reporting on open hernia technique still describe ligating the sac. ${ }^{15,16}$ In all of the above references the sac ligation was either quoted in passing or it was over emphasized as an essential part of the repair. There was, however, very little support for this step and no further clinical or experimental analysis provided.

In the present work, forming Lichtenstein 
repair, invagination and excision of the hernia sac were associated with a similar statistical risk of hernia recurrence surgery as sac ligation.

Recurrence rates have fallen markedly following the introduction of mesh and its current widespread use. In the original description of the Lichtenstein technique, the indirect hernia sac is dissected from the cord to a point beyond the neck of the sac, opened and then returned into the preperitoneal cavity without excision. ${ }^{17-19}$ The sheet of mesh should protect against and hold back any indirect inguinal hernia bulge.

It has been shown that, during abdominal surgery, the peritoneum heals rapidly within 2-3 days, leaving no adhesions, regardless of the size of the defect $^{2}$ and leaving the peritoneum open was not associated with an increased incidence of wound failure. 6,7

The place of non-ligation ${ }^{20-21}$ was well documented by Smedberg et al, ${ }^{5}$ who presented the only prospective and controlled study of either ligation and excision of the sac or excising the sac as deep as possible, then leaving it open without ligation or transfixation. They used both clinical and radiological methods to define recurrence. The study showed that leaving the sac open does not compromise the repair and results in less post-operative pain. Their study has been quoted in some surgical textbooks. ${ }^{21,22}$

In the Shouldice technique the indirect hernia sac is opened, inspected, and then either excised or simply returned into the preperitoneal space. High ligation is considered unnecessary. Thorough dissection and reconstruction of the internal inguinal ring is performed routinely during Shouldice repair, giving recurrence rates of around 1\% in specialized centers but as high as 10-15 $\% 3$ years after surgery in non-specialized units. ${ }^{23,24}$

The sac is not ligated in Rutkow's Mesh Plug repair but inverted. Rutkow recommended that hernia sacs should not be opened for visual inspection because it simply involutes without any problem in few days. He thinks that because peritoneum is a highly sensitive structure ligating the sac does nothing more than produce miniature "peritonitis". This iatrogenic peritonitis is one of the factors contributing to the postoperative discomfort, pain and malaise that accompany suture hernia repairs. The reported recurrence rate in Rutkow's Mesh Plug repair is $0.2 \%{ }^{6}$

High dissection of the sac and not high ligation is the critical factor. High ligation does not influence recurrence rate and may be a cause of increased post-operative pain. ${ }^{10}$ However, high dissection of the sac well up into the retroperitoneum and the freeing of the sac from the edges of the internal ring are important for the prevention of recurrence and allow good exposure of the ring to facilitate the repair. The sac is not ligated in Gilbert's sutureless repair and laparoscopic hernia repairs. ${ }^{25,26}$

Outcomes other than recurrence such as long-term postoperative pain and discomfort are now of greater clinical importance. Recurrence rates are so low after mesh repair that chronic pain is currently the issue of greatest importance following groin hernia repair.

In our series post-operative pain was significantly less when the sac was invaginated or excised than when ligated. Following excision of the hernia sac, pain during convalescence may increase if the peritoneal opening is ligated in open sutured repair. ${ }^{5}$ Similarly, in open mesh repair early postoperative pain has been found to be greater after excision than after invagination of the hernia sac. ${ }^{19}$

Neither of those studies ${ }^{5,19}$ nor the present study showed an important risk of chronic pain after invagination, excision or ligation of the sac. Chronic pain in the inguinal region was reported during follow up after inguinal hernioplasty. The reason for this is the failure to identify and preserve nerve structures during surgery. A recent reviews on nerve management during open hernia repair suggested that nerves should probably be identified to prevent damage caused by sutures placed near the nerves. ${ }^{27,28}$ The mesh frequently causes temporary discomfort during the first year after surgery independent 
Table 1: The demographic characteristics of patients (percentage to patient number).

\begin{tabular}{|l|l|l|l|l|}
\hline & \multicolumn{1}{|c|}{ Group I } & \multicolumn{1}{c|}{ Group E } & \multicolumn{1}{c|}{ Group L } & $\mathrm{f}(\mathrm{p})$ \\
\hline Age (years) & $48.40 \pm 21.01$ & $44.27 \pm 24.67$ & $48.36 \pm 19.90$ & $0.59(0.556)$ \\
\hline Gender & & & & \\
males & $50(32.8 \%)$ & $47(30.9 \%)$ & $52(34.2 \%)$ & \\
Females & 0 & $2(1.31 \%)$ & $1(0.66 \%)$ & \\
\hline Occupation & & & & \\
Manual worker & $33(21.7 \%)$ & $21(13.8 \%)$ & $25(16.4 \%)$ & \\
Intellectual worker & $17(11.1 \%)$ & $27(17.8 \%)$ & $28(18.4 \%)$ & \\
Housewives & & $1(0.66 \%)$ & & \\
\hline Current smoking & $39(25.7 \%)$ & $35(23.0 \%)$ & $41(27.0 \%)$ & \\
Co morbidities & $14(9.2 \%)$ & $18(11.8 \%)$ & $17(11.1 \%)$ & \\
\hline
\end{tabular}

Table 2: The clinical data of patients (percentage to patient number).

\begin{tabular}{|l|l|l|l|l|}
\hline & \multicolumn{1}{|c|}{ Group I } & \multicolumn{1}{|c|}{ Group E } & \multicolumn{1}{c|}{ Group L } & \multicolumn{1}{c|}{$\mathrm{f}(\mathrm{p})$} \\
\hline Number of patients & 50 & 49 & 53 & \\
\hline Number of hernias & 54 & 56 & 57 & \\
\hline Duration of symptoms (months) & $39.32 \pm 21.79$ & $35.14 \pm 22.48$ & $40.24 \pm 22.39$ & $0.75(0.474)$ \\
\hline symptoms & & & & \\
Bulging & $6(3.9 \%)$ & $7(4.6 \%)$ & $10(6.6 \%)$ & \\
Pain & $5(3.3 \%)$ & $7(4.6 \%)$ & $6(3.9 \%)$ & \\
Both & $39(25.6 \%)$ & $35(23.0 \%)$ & $37(24.3 \%)$ & \\
Scrotal hernia & $6(3.9 \%)$ & $5(3.3 \%)$ & $8(5.3 \%)$ & \\
\hline Primary hernia location & & & & \\
Unilateral & $46(37.2 \%)$ & $42(27.6 \%)$ & $49(32.2 \%)$ & \\
Bilateral & $4(2.6 \%)$ & $7(4.6 \%)$ & $4(2.6 \%)$ & \\
\hline Classification of hernia $(\mathrm{Gilbert}, 25)$ & & & & \\
Type I (Normal internal ring) & $14(9.2 \%)$ & $16(10.5 \%)$ & $13(8.6 \%)$ & \\
Type II (Internal ring < $4 \mathrm{~cm})$ & $31(20.4 \%)$ & $34(22.4 \%)$ & $36(23.7 \%)$ & \\
Type III ( Internal ring $>4 \mathrm{~cm})$ & $5(3.3 \%)$ & $6(3.9 \%)$ & $4(2.6 \%)$ & \\
\hline
\end{tabular}

Table 3: Post operative pain.

\begin{tabular}{|l|l|l|l|r|}
\hline Postoperative pain & \multicolumn{1}{|c|}{ Group I } & Group E & Group L & $\mathrm{f}(\mathrm{p})$ \\
\hline Minimal (VAS 0-1) & 11 & 9 & 9 & \\
Mild (VAS 2-3) & 31 & 23 & 26 & \\
Moderate (VAS 4-6) & 5 & 14 & 13 & \\
Severe (VAS 7-10) & 3 & 3 & 5 & \\
Mean Pain Score & $3.04 \pm 2.11$ & $3.98 \pm 2.33$ & $4.06 \pm 2.43$ & $3.07(0.049)$ \\
\hline
\end{tabular}

of the surgical method used. This discomfort at the inguinal region then subsides when the mesh integrates permanently with the abdominal wall.29,30

\section{Conclusion :}

Invagination and excision of the hernia sac do not have adverse effects on repair integrity. They limit the dissection and reduce the morbidity and risk of injury to the spermatic cord and surrounded structures. They are safer 
Table 4 Postoperative complications in both groups (percentage to hernia number).

\begin{tabular}{|l|l|l|l|l|}
\hline Post operative complication & Group I & Group E & Group L & $\mathrm{f}(\mathrm{p})$ \\
\hline Seroma & 0 & $1(0.60 \%)$ & $1(0.60 \%)$ & \\
Superfacial wound infection & $1(0.60 \%)$ & $1(0.60 \%)$ & $2(1.20 \%)$ & \\
Deep wound infection & $1(0.60 \%)$ & 0 & 0 & \\
Postoperative pain & & & & \\
Chronic pain & $3(1.80 \%)$ & $5(3.00 \%)$ & $3(1.80 \%)$ & \\
Recurrence & $3(1.80 \%)$ & $1(0.60 \%)$ & $1(0.60 \%)$ & $0.85(0.429)$ \\
Total number of complications & $8(4.80 \%)$ & $8(4.80 \%)$ & $7(4.20 \%)$ & $0.12(0.887)$ \\
\hline
\end{tabular}

and more appropriate for repair of sliding hernia. Ligation of the hernia sac in inguinal hernia surgery is not only unnecessary and time consuming but also leads to increased postoperative pain. Recurrence rates are statistically unaffected by not ligating the sac.

\section{Reference:}

1- Bassini E: Ueber die Behandlung des Leistenbruches. Archiv fur Klinische Chirurgie 1890; 40: 429-476.

2- Delikoukos S, Lavant L, Hlias G, Palogos K, Gikas D: The role of hernia sac ligation in postoperative pain in patients with elective tension-free indirect inguinal hernia repair: A prospective randomized study. Hernia. 2007; 11(5): 425-428.

3- Gharaibeh KI, Matani YY: To ligate or not to ligate the hernial sac in adults? Saudi Med J 2000; 21(11): 1068-1070.

4- Shafik A: Invagination of the hernial sac stump. Technique for repair of inguinal hernia. Am J Surg 1980; 140(3): 431-436.

5- Smedberg SG, Broomé AE, Gullmo A: Ligation of the hernial sac? Surg Clin North Am 1984; 64(2): 299-306.

6- Yung S, Davies M: Response of the human peritoneal mesothelial cell to injury: An in vitro model of peritoneal wound healing. Kidney Int 1998; 54(6): 2160-2169.

7- Ellis H, Heddle R: Does the peritoneum need to be closed at laparotomy? Br J Surg 1977; 64(10): 733-736.

8- Lichtenstein IL, Shulman AG, Amid PK, Montllor MM: The tension free hernioplasty. Am J Surg 1989; 157(2): 188-193.

9- Rutkow IM, Robbin AW: Tension-free inguinal herniorrhaphy: A preliminary report on the "mesh plug" technique. Surgery 1993; 114(1): 3-8.

10- Abrahamson J: Etiology and pathophysiology of primary and recurrent groin hernia formation. Surg Clin North Am 1998; 78(6): 953-972.

11- Postlethwait RW: Recurrent inguinal hernia. Ann Surg 1985; 202(6): 777-779.

12- Pietri P, Gabrielli F: Recurretn inguinal hernia. Int Surg 1986; 71(3): 164-168.

13- Griffith CA: The Marcy repair revisited. Surg Clin North Am 1984; 64(2): 215-227.

14- Weinstein M, Roberts M: Recurrent inguinal hernia. Follow-up study of 100 postoperative patients. Am J Surg 1975; 129(5): 564-569.

15- Callesen T, Bech K, Andersen J, Nielsen R, Roikjaer $\mathrm{O}$, Kehlet H: Pain after primary inguinal herniorraphy: Influence of surgical technique. J Am Coll Surg 1999; 188: 355-359.

16- Panos RG, Beck DE, Maresh JE, Harford FJ: Preliminary results of a prospective randomized study of Cooper's ligament versus Shouldice herniorrhaphy technique. Surg Gynecol Obstet 1992; 175(4): 315-319.

17- Amid PK, Shulman AG, Lichtenstein IL: Open 'tension-free' repair of inguinal hernias: The Lichtenstein technique. Eur $J$ Surg 1996; 162(6): 447-453.

18- Lichtenstein IL, Shulman AG, Amid PK: Twenty questions about hernioplasty. Am Surg 1991; 57(11): 730-733.

19- Shulman AG, Amid PK, Lichtenstein IL: Ligation of hernial sac. Aneedless step in adult hernioplasty. Int Surg. 1993;78(2):152-153.

20- Lichtenstein IL. Herniorrhaphy: A personal experience with 6,321 cases. Am J Surg 1987; 153(6): 553-559.

21- Abrahamson J: Hernias. In: Maingot's abdominal operations. Schwarts SI, Ellis H, editors. $9^{\text {th }}$ edition. Conneticut: Prentice-Hall International Inc; 1990. p. 215-296.

22- Wantz GE. Abdominal wall hernias. In: Priciples of surgery. Schwartz SI, Shires GT, Spencer FC, Daly GM, Fischer JE, Galloway AC, editors. $17^{\text {th }}$ edition. Volume 2. USA: McGraw-Hill; 1999. p. 1585-1611. 
23- Haapaniemi S, Nilsson E: Recurrence and pain three years after groin hernia repair. Validation of postal questionnaire and selective physical examination as a method of follow-up. Eur J Surg 2002; 168(1): 22-28.

24- Bendavid R: The Shouldice repair: In Abdominal Wall Hernias: Principles and Management. Bendavid R, Arregui M, Flament JB, Phillips EH (eds). Springer: New York, 2001; 370-375.

25- Gilbert AI, Graham MF: Sutureless technique: Second version. Can J Surg 1997; 40(3): 209-212.

26- McCormack K, Wake BL, Fraser C, Vale L, Perez J, Grant A: Transabdominal pre-peritoneal (TAPP) versus totally extraperitoneal (TEP) laparoscopic techniques for inguinal hernia repair: A systematic review. Hernia 2005; 9(2): 109-114.

27- Wijsmuller AR, van Veen RN, Bosch JL, Lange JF, Kleinrensink GJ, Jeekel J, Lange JF: Nerve management during open hernia repair. Br J Surg 2007; 94(1): 17-22.

28- Bartlett DC, Porter C, Kingsnorth AN: A pragmatic approach to cutaneous nerve division during open inguinal hernia repair. Hernia 2007; 11(3): 243-246.

29- da Costa PM: Complications and recurrences after different types of hernia repair: How to deal with it? Acta Chir Belg 2009; 109(1): 36-41.

30- Bay-Nielsen M, Nilsson E, Nordin P, Kehlet $\mathrm{H}$ : Chronic pain after open mesh and sutured repair of indirect inguinal hernia in young males. Br J Surg 2004; 91(10): 1372-1376. 